\title{
THE APPLICATION OF THE EQUIVALENT ANNULUS WIDTH CONCEPT IN THERMAL DIFFUSION SEPARATIONS
}

\author{
J. J. B. ROMERO $\dagger$ and J. DE D. R. S. PINHEIRO $\ddagger$ \\ Departamento de Engenharia Química, Universidade de Lourenco Marques, Mozambique
}

(Received 15 January 1975; accepted 16 May 1975)

\begin{abstract}
The equivalent annulus width concept is used to characterize a small commercial thermogravitational thermal diffusion column and its validity checked experimentally by separating batchwise in the column mixtures of $n$-heptane-benzene with different initial concentrations. The equation of Ruppell and Coull was used to analyse the data in the short separation times range and determine the equivalent annulus width.

Good agreement was obtained between the experimental and predicted time-separation curves when using the equivalent annulus width value and on averaged value of the thermal diffusion constant. A new method is presented for the simultaneous determination of the equivalent annulus width and the thermal diffusion constant of a binary mixture from a single set of experimental data.
\end{abstract}

\section{INTRODUCTION}

The mathematical theory of the thermogravitational thermal diffusion columns leading to the transport equation, as first developed by Furry, Jones and Onsager [1], involves a considerable number of simplifying assumptions regarding the column geometry, the hydrodynamics in the annular space and the physical and transport properties, besides the usual mathematical simplications and the quasi-stationary assumption.

The extensive research in the field shows that in general the above theory is adequate for many practical situations, though significant discrepancies have been reported regarding the theoretical and the experimental functional relationship between the transport coefficients and the annular width $2 \omega$.

Another source of difficulty is the value of the thermal diffusion constant: for a given mixture, temperature and composition, the reported value of this constant may vary by an order of magnitude, specially when one compares values obtained in horizontal cells with those obtained from vertical columns data. Also the variation of the thermal diffusion constant, $\alpha$, with the temperature, though relatively small, should not always be neglected [2] and its dependence on the concentration is appreciable [3-7]; facts which are ignored in the majority of published papers. This has led some authors [8-11] to consider $\alpha$ as a parameter to be determined experimentally.

This practice however, makes the comparison of the published results rather difficult, particularly as regards the fitting of experimental separation curves with the theoretical predictions. Similar considerations could be made about the ordinary diffusion coefficient and other physical properties, but it should be emphasised that the difficulties above mentioned do not invalidate the theory;

†Current address: Universidade do Minho, Largo do Paço, Braga, Portugal.

$\ddagger$ Current address: Chemical Engineering Department, Birmingham University, Edgbaston, Birmingham B15 2TT, U.K. for instance, the functional dependence of $\alpha$ with concentration can be accommodated in the transport equation, it merely making the subsequent integrations rather more cumbersome.

The validity of the relationship relating the transport coefficient to the annulus width, $2 \omega$, has however, been questioned. In a paper published in 1963, Hoffman and Emery[12] correlated their own results and those obtained by other authors [5, 13-15] and concluded that the deviations observed among the several sets of experimental data and between these and the theory were not accidental but statistically significant; they attributed these deviations to the difficulty of measuring accurately $(2 \omega)$. Romero[16] and Romero and Bott[17] using the "floating column" method, studied the performance of several sections of a carefully constructed thermogravitational column and showed that the deviations found resulted from the irregularity of the annular space along the length of the column. By comparing the hydrodynamic behaviour of truly concentric and eccentric columns, they note that "... the existence of eccentricity means that the convection currents increase and therefore that their remixing effect also increases relatively to the thermal diffusion separation. The degree of separation as well as the relaxation time will thus decrease and it can be said that the physical situation becomes equivalent to the use of a thermal diffusion column with a larger annulus width, the other parameters being kept constant". The same authors stress however, that ". . . the real situation is more complex since it is not likely that the eccentricity be uniform along the column and therefore the flow rate varies also in the vertical direction. The problem then becomes similar to the forgotten effect, as it was interpreted by Korchinsky and Emery".

Korchinsky and Emery [9] have indeed demonstrated that small irregularities in wall spacing could create concentration reversal regardless of the sign of the forgotten effect. Their work, explains the anomalous behaviour referred in some papers $[18,19]$ as caused by geometrical non-uniformity and points out its formal 
similarity with the forgotten effect. It is worth nothing here that Korchinsky and Emery concluded, in their study of the forgotten effect, that it could be important in transient batch operation, particularly for very narrow annulus, but has no influence at the steady state.

It can therefore be concluded that the irregularities in the annular space have the following implications:

(i) A decrease of the equilibrium degree of separation and, in principle of the relaxation time, due to the fact that the hydrodynamics of the column lead to higher values of the vertical convection currents.

(ii) In the transient state the irregularities cause a behaviour of the system similar to the variations of density with concentration, with the existence of transverse flows within the column and the consequent variation of longitudinal fluxes.

From a practical viewpoint, the above results mean that, even if a rigorous profile of the annulus width along the length of a commercial column could be measured, the theoretical analysis of that particular column would be too complex to warrant the effort, keeping in mind that the results would not be valid for any other column; in fact it is not to be generally expected that any two columns have identical profiles for their annulus widths. To overcome this difficulty, Bott and Romero[17] have introduced, the concept of the "equivalent annulus width", which they defined as the annulus width of a geometrically perfect column achieving the same degree of separation as the actual imperfect column, all the other parameters being the same. These authors showed how the value of the equivalent annulus width can be determined from unsteady state experimental data using the equation of Ruppel and Coull, but their own data was insufficient to test the usefulness of the concept.

It is the purpose of the present work to continue this line of research by using the equivalent annulus width concept to predict the behaviour of a commercial column.

\section{THE TRANSPORT EQUATION AND ITS SOLUTIONS}

A good review of the derivation and integration of the transport equation for batch thermogravitational columns closed at both ends has recently been presented by Romero[10] who discussed in detail the assumptions involved in the mathematical development. Here, only the equations relevant to this work are referred.

The basic transport equation derived by Furry et al. [1] and de Groot[20] has the form

$$
\tau=H c(1-c)-K \frac{\partial c}{\partial z}
$$

where the transport coefficients are defined by

$$
H=\frac{\alpha \beta \rho g B(\Delta T)^{2}(2 \omega)^{3}}{6 ! T_{m} \eta}
$$

tThe critical nature of the annulus width and its effect on separation can be appreciated by eqns (6) and (7) and, it is interesting to point out that many experimental research have been carried out in columns with reported annulus widths of about $0.03 \mathrm{~cm}[8,10,21-26]$. This means that deviations of only $0.003 \mathrm{~cm}$, i.e. $10 \%$ of the actual value of $(2 \omega)$ imply an error of $40 \%$ in the computed value of $\lambda$.

$$
\begin{gathered}
K=K_{c}+K_{d} \\
K_{c}=\frac{\beta^{2} \rho g^{2} B(\Delta T)^{2}(2 \omega)^{7}}{9 ! D \eta^{2}} \\
K_{d}=\rho D B(2 \omega) .
\end{gathered}
$$

For steady-state conditions $(\tau=0)$ the integration of eqn (1) yields the following solution, expressed in terms of the separation factor, $\phi$

$$
\phi_{\infty}=\left[\frac{c_{B}\left(1-c_{T}\right)}{c_{T}\left(1-c_{B}\right)}\right]_{\infty}=\mathrm{e}^{\lambda}
$$

where $\lambda$, the dimensionless length of the column, is given by $\dagger$

$$
\begin{aligned}
\lambda & =\frac{H L}{K}=\frac{H L}{K_{c}} \frac{1}{\left(1+K_{d} / K_{c}\right)} \\
& =\frac{504 \alpha D \eta L}{\beta g T_{m}(2 \omega)^{4}} /\left[1+5670\left(\frac{D \eta}{\omega^{3} \beta g(\Delta T)}\right)^{2}\right]
\end{aligned}
$$

For unsteady-state conditions, the solutions of the transport equation so far obtained involve an infinite series. Hoffman [28] and Majumdar[27] found a solution that converges rapidly for large values of time. In fact, Hoffman and Emery [2] using numerical analysis techniques concluded that after a time $t \geqslant 0.3 t_{r}$ the terms of the series beyond the first can be neglected with an error less than $1 \%$. In this case, the concentration difference between the extremities of the column, $\Delta$ is simply given by

$$
\Delta=\Delta_{\infty}\left(1-k_{3} \mathrm{e}^{-t / t_{4}}\right)
$$

where $\Delta_{\infty}$-the steady-state separation-, $t_{r}$-the relaxation time-and the coefficient $k_{3}$ are determined by the following expressions

$$
\begin{gathered}
\Delta_{\infty}=\frac{\left(\mathrm{e}^{c_{0} \lambda}-1\right)\left(\mathrm{e}^{\lambda}-\mathrm{e}^{c_{0} \lambda}\right)}{\mathrm{e}^{c_{0} \lambda}\left(\mathrm{e}^{\lambda}-1\right)} \\
t_{r}=\frac{\mu K}{H^{2}} \cdot \frac{1}{\frac{1}{4}+\frac{\pi^{2}}{\lambda^{2}}}=\frac{10}{7} \frac{T_{m}^{2}(2 \omega)^{2}}{(\Delta T)^{2} \alpha^{2} D} \cdot \frac{1}{\frac{1}{4}+\frac{\pi^{2}}{\lambda^{2}}} \\
k_{3}=\frac{2 c_{0}\left(1-c_{0}\right) \pi^{2}\left(1+\mathrm{e}^{-b_{0} \lambda}\right)\left(\mathrm{e}^{\lambda}-1\right)\left(\mathrm{e}^{c_{0} \lambda}+\mathrm{e}^{\lambda / 2}\right)}{\lambda^{3}\left(b_{0}{ }^{2}+\frac{\pi^{2}}{\lambda^{2}}\right)\left(\frac{1}{4}+\frac{\pi^{2}}{\lambda^{2}}\right)\left(\mathrm{e}^{c_{0} \lambda}-1\right)\left(\mathrm{e}^{\lambda}-\mathrm{e}^{c_{0} \lambda}\right)} \simeq \frac{8}{\pi^{2}} .
\end{gathered}
$$

A solution for unsteady-state conditions that converges rapidly for small values of time was proposed by Ruppel and Coull[29]. For sufficiently small values of the time variable, the terms of the infinite series beyond the first can be neglected. In this case the Ruppel and Coull solution [30] is reduced to

$$
\Delta=4 c_{0}\left(1-c_{0}\right) \sqrt{\frac{\theta}{\pi}}
$$


where $\theta$, the dimensionless time is defined by

$$
\theta=\frac{H^{2}}{\mu K} t=\frac{7}{10}\left(\frac{\alpha(\Delta T) D^{1 / 2}}{T_{m}(2 \omega)}\right)^{2} \cdot t
$$

The range of time to which eqn (12) applies is such that [29]

$$
\theta \leqslant 0 \cdot 05|\lambda|^{1.82}
$$

\section{APPARATUS AND EXPERIMENTAL CONDITIONS}

The commercial column used $\dagger$ whose main dimensions are shown in Table 1 is identical to others described in the literature $[8,18]$ apart from a few modifications specified when ordering the column. These included the provision of additional thermocouples in the hot wall and a redesign of the sampling ports whose caps were adapted to take syringes and provided with hollow stems to reduce the dead volume in the outlet nipples.

Table 1. Basic dimensions of the column (as indicated by the manufacturer)

\begin{tabular}{lc}
\hline I.D. outer cylinder: & $1.654 \mathrm{~cm}$ \\
O.D. inser cylindex: & $1.593 \mathrm{~cm}$ \\
Annulus width, $\left(\mathrm{r}_{2}-\mathrm{r}_{1}\right):$ & $0.0305 \mathrm{~cm}$ \\
Length: & $182.56 \mathrm{~cm}$ \\
\hline
\end{tabular}

The experimental technique was analogous to that described by Bott and Romero[17] except the rate of sampling which was zero (1 sample per run) in accordance with the recommendations of Vichare and Powers [31].

The temperature difference between the hot and cold walls was $(15 \pm 0.5)^{\circ} \mathrm{C}$ and the mean temperature $(27 \pm$ 2) ${ }^{\circ} \mathrm{C}$. The vertical temperature gradient was kept below $0.5^{\circ} \mathrm{C}$.

Mixtures of $n$-heptane-benzene were used in this work since there is considerable information in the literature about their physical and transport properties [33] and has become almost a "standard" system for the study of the performance of thermal diffusion equipment. The purification of the solvents and methods of analysis are described elsewhere [34].

\subsection{Experimental results}

\section{RESULTS AND DISCUSSION}

Three feed concentrations were investigated in the thermal diffusion column, namely, $c_{0}=0.25, c_{0}=0.50$, $c_{0}=0.75$. For each feed mixture the evolution of concentration was determined experimentally, the whole time range of interest being virtually covered, i.e. from very short times to times where the concentration profiles are very near equilibrium. The experimental points obtained are shown in Figs. 1 and 3 as plots of $\Delta$ against $t$ and $\sqrt{t}$.

\subsection{Equivalent annulus width evaluation}

As in most practical cases, the term $\left(K_{d} / K_{c}\right)$ in eqn (7)

†Supplied by M. Fink Co., Cleveland, Ohio, U.S.A.

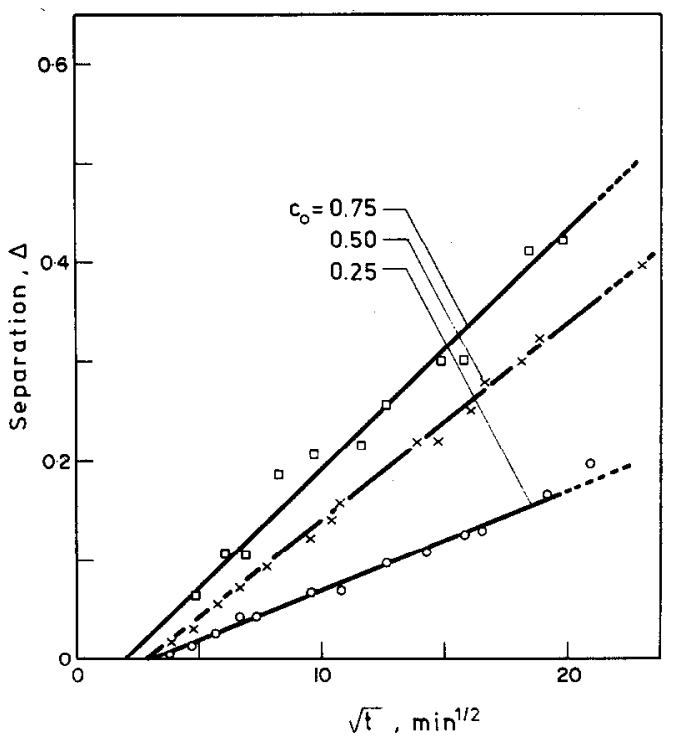

Fig. 1. Separation against the square root of time in the short experimental time range where the Ruppel and Coull equation applies.

is very small and can be neglected as compared to unity with an error less than $1 \%$. The equation of Ruppel and Coull can, then, be written more simply as

$$
\Delta=\frac{4 c_{0}\left(1-c_{0}\right)(\Delta T)}{45 T_{m}} \sqrt{\frac{2835}{2 \pi}} \frac{\alpha}{2 \omega} \sqrt{t}
$$

or

$$
\Delta=m \frac{\alpha}{2 \omega} \sqrt{t}
$$

in which " $m$ " represents the term between square brackets in eqn (15) depending only on the properties of the mixture and the experimental conditions, and, therefore, can be evaluated accurately.

In the range of time where the Ruppel and Coull equation applies, the plot of $\Delta$ vs $\sqrt{t}$ must, thus, be a straight line of slope " $s$ " such that

$$
\frac{\alpha}{2 \omega}=\frac{s}{m}
$$

Statistical analysis using the least squares method showed, indeed, a good linear regression between $\Delta$ and $\sqrt{t} t$ for all the three feed compositions. A summary of the results is shown in Table 2 and Fig. 1 and it is seen that the intersept $\Delta_{0}$ for $t=0$ is not zero as predicted by eqn (15). The explanation was given by Madariaga et al.[35] who showed that the non-zero intersept was due to the very small "reservoirs" that build up at the extremities of the column without, however, affecting the value of the slope, s.

Equation (17) can, now, be used for two different objectives as suggested by Bott and Romero[17]:

(A) To determine $\alpha$ knowing ( $2 \omega$ ) 
Table 2. Linear regression analysis $\left(\Delta=s \sqrt{t}+\Delta_{0}\right)$

\begin{tabular}{|c|c|c|c|}
\hline $\begin{array}{l}\text { Feed } \\
\text { composition }\end{array}$ & $\begin{array}{l}\text { slope, } s .10^{2} \\
\left(\min ^{-1}\right)\end{array}$ & $\begin{array}{c}\text { Intersept, } \\
\Delta_{0} \cdot 10^{2} \\
\end{array}$ & $\begin{array}{l}\text { Correlation } \\
\text { coefficient, } r\end{array}$ \\
\hline 0.25 & 1.07 & -3.59 & 0.989 \\
\hline 0.50 & 2.17 & -7.18 & 0.994 \\
\hline 0.75 & 2.32 & -4.46 & 0.971 \\
\hline
\end{tabular}

(B) To determine ( $2 \omega)$-the equivalent annulus widthknowing $\alpha$.

In case $A$ the column is assumed to be perfect, i.e. $(2 \omega)=\left(r_{2}-r_{1}\right)=0.0305 \mathrm{~cm}$ (cf. Table 1). Substituting then $(2 \omega)$, " $\mathrm{m}$ " and "s" by their values in eqn (17), the following values for $\alpha$ are obtained $\dagger$

$$
\begin{aligned}
& \alpha_{0.25}=0.42 \\
& \alpha_{0.50}=0.69 \\
& \alpha_{0.75}=1.07 .
\end{aligned}
$$

These values of $\alpha$ are quite low in comparison with others reported in the literature obtained also in thermogravitational columns (see Table 3) therefore suggesting that the value of $(2 \omega)$ must be higher than $0.0305 \mathrm{~cm}$, or, in other words, that an "equivalent annulus width" must be considered.

Table 3. Values of the thermal diffusion constant for the $n$-benzene-heptane system

\begin{tabular}{cccc}
\hline & \multicolumn{3}{c}{$|0|$} \\
\cline { 2 - 4 } Reference & $c_{0}=0.25$ & $c_{0}=0.50$ & $c_{0}=0.75$ \\
\hline (32) & - & $1.19-1.28$ & - \\
(8) & - & 1.2 & - \\
(10) & - & 1.02 & - \\
(7) & 0.88 & 1.19 & 1.67 \\
(6) & 0.99 & 1.36 & 1.89 \\
(4) & 0.86 & 1.27 & 1.75 \\
(3) & 1.0 & 1.24 & 1.45 \\
(5) & 0.8 & 1.35 & 1.89 \\
Present work & $0.71 \pm 0.07$ & $1.16 \pm 0.09$ & $1.84 \pm 0.10$ \\
(new method) & & & \\
\hline
\end{tabular}

Before case B can be examined it is, however, necessary to find a reasonably accurate value of $\alpha$. The dispersion of the reported values is, though, quite appreciable which renders the choice somewhat arbitrary. In this case, by consideration of the average and median values of the distribution of $\alpha_{0.50}$ in Table 3 the following value was chosen:

$$
\alpha_{0.50}=1 \cdot 24
$$

†According to the usual convention, the thermal diffusion constant, $\alpha$, for the system $n$-heptane-benzene is negative. However, for simplicity the minus sign is omitted.
The values of $\alpha_{0.25}$ and $\alpha_{0.75}$ are determined through the relations below derived from eqn (17)

$$
\begin{aligned}
& \alpha_{0.25}=\alpha_{0.50} \frac{s_{0.25}}{s_{0.50}} \frac{m_{0.50}}{m_{0.25}}=0.74 \\
& \alpha_{0.75}=\alpha_{0.50} \frac{s_{0.75}}{s_{0.50}} \frac{m_{0.50}}{m_{0.75}}=1.77
\end{aligned}
$$

The computation of $\alpha_{0.25}$ and $\alpha_{0.75}$ in this manner is justified not only by the comparatively small number of points that constitute their distributions (see Table 3) but principally because the procedure implies that the value of the equivalent annulus width determined through eqn (17) is the same irrespective of the feed-composition data used. Thus,

$$
2 \omega=\frac{m \alpha}{s}=0.055 \mathrm{~cm} .
$$

It is seen that this value of $(2 \omega)$ is significantly different from the value of $2 \omega=0.0305 \mathrm{~cm}$ indicated by the manufacturer (Table 1).

The corresponding values of $\alpha$ and $(2 \omega)$ for cases A and $B$ are summarized in Table 4 for the three feed compositions used.

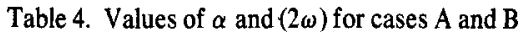

\begin{tabular}{ccc}
\hline \multirow{2}{*}{$c_{0}$} & \multicolumn{2}{c}{$a$} \\
\cline { 2 - 3 } & $2 \omega=0.0305 \mathrm{~cm}$ & $2 \omega=0.055 \mathrm{~cm}$ \\
\hline 0.25 & 0.42 & 0.74 \\
0.50 & 0.69 & 1.24 \\
0.75 & 1.07 & 1.77 \\
\hline
\end{tabular}

For each feed composition it is now possible to determine the theoretical separation curves given by eqn (8)-the Hoffman and Emery equation-for cases $A$ and $B$ and compare them with the experimental separation curve. In doing so, it is found that for all values of $c_{0}$ a much better agreement is obtained in case $B$ as illustrated in Fig. 2 for $c_{0}=0 \cdot 50$. (For the other feed concentrations, $c_{0}=0.25$ and $c_{0}=0.75$ the relative position of the curves is identical.)

The good fit obtained between the experimental points and the theoretical separation curve calculated using an equivalent annulus width value of $2 \omega=0.055 \mathrm{~cm}$ and an average $\alpha$ in eqn (17) suggests two important observations:

(1) The equivalent annulus width is an adequate means of characterizing the performance a non-perfect column.

(2) The equivalent annulus width determined in the short experimental times range through the Ruppel and Coull equation can be used to describe the performance of the column at any higher value of the time variable, i.e. the equivalent annulus width does not change as the separation proceeds. 


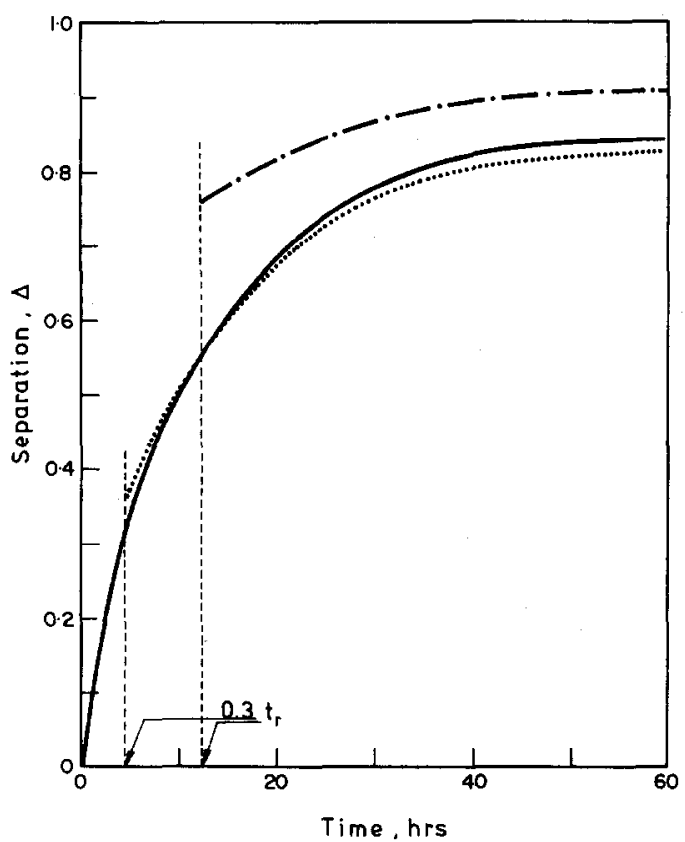

Fig. 2. Comparison between the theoretical and experimental separation-curves when using the equivalent annulus width (case B) or $2 \omega=r_{2}-r_{1}$ (case A): ——, experimental; - - - eqn (8), case $A ; \cdots \cdots \cdots$, eqn $(8)$, case $B$.

\section{A NEW METHOD FOR THE SIMULTANEOUS

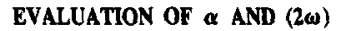

The process of evaluating the equivalent annulus width presented in the preceding section is not entirely satisfactory since it requires the use of an accurate value of the thermal diffusion constant, $\alpha$, which is still an "open question". If, however, the final observations of the previous section are considered, it is possible to derive a new method for computing the equivalent annulus width independently of $\alpha$ and, simultaneously, to obtain the mean value of the thermal diffusion constant on the experimental conditions used.

In fact, the apparent non-dependency of the equivalent annulus width on separation suggests that its value can be determined either in the transient "Ruppel and Coull" period (as done before) or in any other region of the separation, namely, the steady-state region in which minor variations on $(\Delta T)$ or the existence of the so-called "forgotten effect" do not affect the separation.

The new method requires the experimental determination of the steady-state composition at the extremities of the column which will enable the evaluation of the parameter $\lambda$ through either eqn (6) or (9).
The defining equation of $\lambda-$ eqn (7)-can be written in a form similar to eqn (17) as

$$
\frac{\alpha}{(2 \omega)^{4}}=\frac{\lambda}{n}
$$

where

$$
n=\frac{504 D \eta L}{\beta g T_{m}}
$$

is independent of both $\alpha$ and $(2 \omega)$ and may be evaluated separately with reasonable accuracy.

Dividing, now, eqn (17) by eqn (25)

$$
\frac{\frac{\alpha}{2 \omega}}{\frac{\alpha}{(2 \omega)^{4}}}=(2 \omega)^{3}=\frac{n s}{m \lambda}
$$

and

$$
2 \omega=\left(\frac{n s}{m \lambda}\right)^{1 / 3} .
$$

Equation (28) allows, thus, the determination of the equivalent annulus width independent from $\alpha$, and it is worth noting that the error in $(2 \omega)$ is one third of the error involved in the evaluation of $(2 \omega)^{3}$ in eqn (27).

The thermal diffusion constant may now be determined through eqn (17) using the value of $(2 \omega)$ from eqn (28)

$$
\alpha=\frac{s}{m} \cdot\left(\frac{n s}{m \lambda}\right)^{1 / 3} .
$$

In Tables 5 and 6 the application of the new method to the experimental results for $c_{0}=0.25,0.59$ and 0.75 is presented. It is apparent the good agreement existing between the data for the three different feed compositions and the satisfactory agreement between the values of $\alpha$ obtained and those reported on Table 3.

The theoretical curves given by eqn (8) for $2 \omega=$ $0.052 \mathrm{~cm}$ and the most probable value of $\alpha$ of Table 6 are plotted in Fig. 3 together with the experimental points obtained. It is seen that the agreement is good.

\section{CONCLUSIONS}

It is thought that the following conclusions can be derived from the present work.

(i) The applicability of the Ruppel and Coull equation in the short experimental times range is confirmed.

(ii) The equivalent annulus width concept seems to be a valid way of characterising the effective distance between

Table 5. Experimental values

\begin{tabular}{ccccc}
\hline$\varepsilon_{0}$ & $\begin{array}{c}2.10^{2} \\
(\min -1),\end{array}$ & $\left(c_{B}\right)_{\infty}$ & $\left(c_{\mathrm{T}}\right)_{\infty}$ & $A_{\sigma}$ \\
\hline 0.25 & $1.07 \pm 0.04$ & $0.60 \pm 0.05$ & $0.04 \pm 0.01$ & $0.61 \pm 0.03$ \\
0.50 & $2.17 \pm 0.05$ & $0.95 \pm 0.02$ & $0.08 \pm 0.02$ & $0.88 \pm 0.04$ \\
0.75 & $2.32 \pm 0.02$ & $0.97 \pm 0.02$ & $0.06 \pm 0.02$ & $0.91 \pm 0.01$ \\
\hline
\end{tabular}


Table 6. Calculated values

\begin{tabular}{ccccccc}
\hline co & $0.10^{3}$ & $0.10^{2}$ & $\lambda$ & $(2 \omega)^{3} \cdot 10^{6}$ & $2 \omega(\mathrm{cm})$ & $|\alpha|$ \\
\hline 0.25 & 0.801 & 4.11 & $4.0 \pm 0.3$ & $138 \pm 14$ & $0.0520 \pm 0.0025$ & $0.71 \pm 0.07$ \\
0.50 & 0.974 & 3.56 & $5.5 \pm 0.4$ & $145 \pm 15$ & $0.0525 \pm 0.0025$ & $1.16 \pm 0.09$ \\
0.75 & 0.659 & 3.18 & $9.6 \pm 0.5$ & $120 \pm 16$ & $0.050 \pm 0.0025$ & $1.84 \pm 0.10$ \\
\hline
\end{tabular}

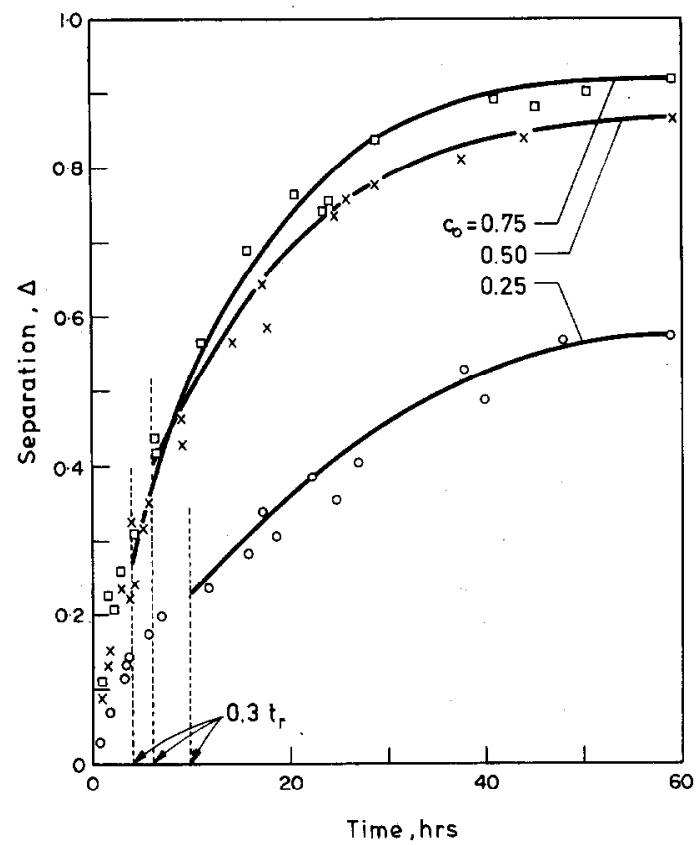

Fig. 3. Experimental points and the theoretical separation-curves for the pairs of values $(\alpha, 2 \omega)$ evaluated through the new method.

the hot and cold walls of a thermal diffusion column both in the steady and unsteady state regimes. However more experimental work in particular with a variety of mixtures is needed to confirm this conclusion.

(iii) Assuming that the equivalent annulus width is indeed an adequate parameter for the characterization of a thermal diffusion column, a simple method is available for the computation of this parameter and of the thermal diffusion constant of a mixture, using a single set of experimental data relating the degree of separation to time.

Acknowledgements-The authors would like to thank Dr. T. R. Bott, University of Birmingham, England, for his comments on the manuscript of this paper, and express their gratitude to Instituto de Alta Cultura, Lisboa, for the provision of a grant which made possible this work.

\section{NOTATION}

$B$ column width in the Y-direction

$b_{0} \quad 1 / 2-c_{0}$

$c$ mole fraction of specified component (benzene)

$c_{0}$ original mole fraction

$D$ diffusion coefficient

$g$ acceleration due to gravity

$H$ transport coefficient due to thermal diffusion (eqn 2)
$K$ transport coefficient to allow for remixing effects (eqn 3)

$K_{c}, K_{d}$ transport coefficients to allow for remixing by correction and ordinary diffusion, respectively (eqns 4, 5)

$k_{3}$ dimensionless parameter (eqn 11)

$L$ length of column

$m$ coefficient defined by (eqns 15,16 )

$n$ coefficient defined by (eqn 26)

$s$ slope of the curve $\Delta$ vs $\sqrt{t}$ defined by (eqn 17)

$T$ mean temperature inside the annulus

$t$ time

$t_{\text {r }}$ relaxation time, (eqn 10)

$z$ vertical coordinate

Greek symbols

$\alpha$ thermal diffusion constant (thermal diffusion factor)

$\beta$ coefficient of thermal expansion

$\Delta$ mole fraction difference between the column ends

$\Delta_{\infty}$ mole fraction difference between the column ends at steady state, (eqn 9)

$\Delta T$ temperature difference between walls

$\phi$ separation factor defined by (eqn 6)

$\theta$ dimensionless time $=H^{2} t / \mu K$

$\eta$ viscosity

$\lambda$ dimensionless length of column $=H L / K$

$\mu$ mass or moles per unit length of column $(\mu=2 \omega B \rho)$

$\rho$ molar density

$\tau$ total transport of specified component in the vertical direction

$\omega$ half distance between hot and cold surfaces

\section{Subscripts}

$T$ top

$B$ bottom

$\infty$ equilibrium (steady-state)

$m$ average

\section{REFERENCES}

[1] Furry W. H., Jones R. C. and Onsager L., Phys. Rev. 193955 1063.

[2] Saxton R. L. and Drickamer H. G., J. Chem. Phys. 195422 1287.

[3] Bott T. R. and Khoo Y. K., Trans. Instn. Chem. Engrs. 1967 45 T115.

[4] Melpolder F. W., Brown R. A., Walshall T. A., Doherty W. and Young W. S., Anal. Chem. 1954261904.

[5] Heines T. S., Larson O. A. and Martin J. J., Ind. Engng Chem. 1957 49(11) 1911. 
[6] Demichowicz-Pigoniowa J., Mitchell M. and Tyrrell H. J., J. chem. Soc. (A), 1971307.

[7] Korsching H., Z. Naturforsch. $196924 a 444$.

[8] Powers J. E., Ind. Engng Chem. 1961 53(7) 577.

[9] Korchinsky W. J. and Emery A. H., A.I.Ch.E. Jl 1967 13(2) 224.

[10] Chrownover C. F. and Powers J. E., A.I.Ch.E. Jl 1962 8(2) 166.

[11] Sanchez V., Mahenc J. and Sagnes M., Chimie et Industrie Génie Chimique 1970 103(18) 2407.

[12] Hoffman D. T. Jr and Emery A. H. Jr., A.I.Ch.E. Jl 1963 9(5) 653.

[13] Powers J. E., Wilke C. R., A.I.Ch.E. Jl 19573213.

[14] Longmire D. R., A.I.Ch. E. $J 19606304$.

[15] Powers J. E., Proc. of the Joint Conf. on Thermodynamic and Transport Properties of Fluids, p. 198. Institution of Mechanical Engineers, London, 1957.

[16] Romero J. J. B., Ph.D. Thesis, University of Birmingham (1967); also Rev. Física, Química e Engenharia (ULM) 1970 2 (Serie A) 1

[17] Bott T. R. and Romero J. J. B., Trans. Instn. Chem. Engrs. $196947 \mathrm{~T} 166$.

[18] Jones A. L. and Milberger E. C., Ind. Engng Chem. 195345 2689.

[19] Prigogine I., Brouckere L. and Amand R., Physica 195016 577851.
[20] de Groot S. R., The Soret Effect. North-Holland, Amsterdam, 1945.

[21] Begeman C.R. and Cramer P.L., Ind.Engng Chem. 195547202

[22] Abelson P. H. and Hoover J. I., In Proc. Intern. Symp. on Isotope Separation, p. 483. North-Holland, Amsterdam, 1957.

[23] de Maria F. and Benenati R. F., Ind. Engng Chem. 19685063.

[24] Brock, J. R., Chem. Engng Sci. 196113207.

[25] Crockett, W. E., Separation of liquids by thermal diffusion. Ph.D. Thesis, University of West Virginia, 1962.

[26] Grigor'ev, V. B., Smirnov, N. T. Zh. Prikl. Khim. 196336 2030.

[27] Majumdar, S. D., Phys. Rev. 195181844.

[28] Hoffman D. T. Jr., Thermal diffusion in liquids. Ph.D. Thesis, University of Kansas, 1962.

[29] Ruppel, T. C., Coull, J., I\&EC Fund. 1964 3(4) 368.

[30] Romero, J. J. B., I\&EC Fund. 19665287.

[31] Vichare, G. G., Powers, J. E., A.I.Ch.E. Jl 1961 7(4) 650.

[32] Trevoy, D. J. and Drickamer H. G., J. chem. Phys. 194917 1120.

[33] Romero J. J. B. Rev. Física, Química e.Engenharia (ULM) 19702 (Serie A) 1.

[34] Pinheiro J. D. S., Pinheiro M., Romero J. J. B., Rev. Física, Química e Engenharia (ULM) 19735 (Serie A) 1.

[35] Madariaga J. A., Alvarez D. G. and Saviron J. M. Revista Acad. Ciencias Exactas Fis-Quim. y Naturales-Zaragoza 19702579. 